(205)

\title{
Screening of Fungi Capable of Degrading Lignin from Decaying Woods in Dry and Intermediate Zone Forests of Sri Lanka
}

\author{
Daraniyagala S.R.A.S. and Attanayake R.N.* \\ Department of Botany, University of Kelaniya, Sri Lanka \\ *renuka@kln.ac.lk
}

\begin{abstract}
Lignin is the third main constituent in wood and is a complex macromolecule formed by the polymerization of three phenylpropane monomers. It is resistant to degradation due to its phenyl propane monomers in the structure and the recalcitrant linkages between them. Therefore, finding microorganisms capable of breaking down lignin is of great interest in biotechnological research specially in biofuel technology. This research was initiated with the aim of isolating strong lignin degraders from the decaying wood pieces obtained from the dry and intermediate zone forests. It was hypothesized that the fungal species found in dry and intermediate zone forests are strong lignin degraders since these forests are rich in hard wood bearing plant species. White rot fungi were isolated into pure cultures from decaying wood pieces showing white rot symptoms on hard wood pieces. In addition, fungal fruiting bodies were also selected whenever possible. Total of 35 pure fungal cultures were isolated and used for further analysis. Each isolate was grown on minimal agar amended with wood powder (mango+burutha powder in 1:1 ratio) and colony diameter was measured after 3 days. Three class of growths, fast growers $(6-9 \mathrm{~cm})$, intermediate growers $(3-6 \mathrm{~cm})$ and slow growers $(0-3$ $\mathrm{cm})$, were tentatively identified based on their colony diameter on wood agar media after three days. Twelve were fast growers, 5 were intermediate growers and the rest were slow growers. Since laccase is one of the important enzymes required for the lignin degradation, the ability to produce laccases by these fungi was estimated by observing the ability to change the color on guaiacol amended PDA. Only three out of 12 fast growers and 4 out of 5 intermediate growers were able to change the color on guaiacol containing media indicating that these isolates were potential wood decaying fungal species with the ability of laccase production. Most of the strong guaiacol producers were basidiomycetes and isolate characterization is being carried out using molecular and morphological methods.
\end{abstract}

Keywords: Lignin degrading, Dry zone forest, Wood decay, White root fungi

Proceedings of the International Forestry and Environment Symposium 2016, Department of Forestry and Environmental Science, University of Sri Jayewardenepura, Sri Lanka. 